\title{
A!
}

This is an electronic reprint of the original article.

This reprint may differ from the original in pagination and typographic detail.

Abe, Aki; Sauerland, Sami; Koskinen, Ari

\section{Highly Enantioselective Conjugate Addition of Thiols Using Mild Scandium Triflate Catalysis}

Published in:

Journal of Organic Chemistry

DOI:

$10.1021 / \mathrm{jo0} 040492 \mathrm{z}$

Published: 01/01/2007

Document Version

Peer reviewed version

Please cite the original version:

Abe, A., Sauerland, S., \& Koskinen, A. (2007). Highly Enantioselective Conjugate Addition of Thiols Using Mild Scandium Triflate Catalysis. Journal of Organic Chemistry, 72(14), 5411-5413. https://doi.org/10.1021/jo070492z

This material is protected by copyright and other intellectual property rights, and duplication or sale of all or part of any of the repository collections is not permitted, except that material may be duplicated by you for your research use or educational purposes in electronic or print form. You must obtain permission for any other use. Electronic or print copies may not be offered, whether for sale or otherwise to anyone who is not an authorised user. 


\section{Highly Enantioselective Conjugate Addition of Thiols Using Mild Scandium Triflate Catalysis}

Aki M. M. Abe, Sami J. K. Sauerland, and Ari M. P. Koskinen*

Laboratory of Organic Chemistry, Helsinki University of Technology, P.O.B. 6100, FI-02015 TKK, Finland

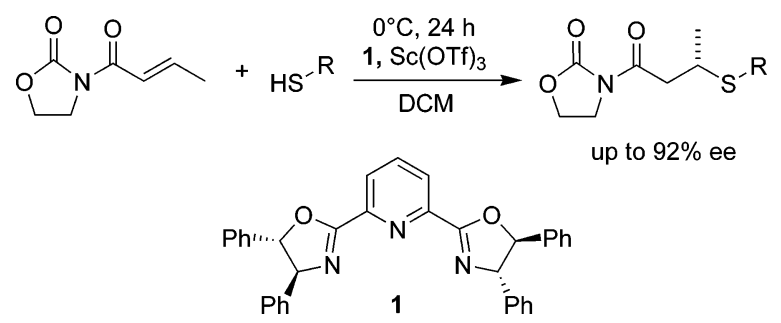

A Sc complex of $(4 S, 5 S)$-diphenyl PYBOX 1 was found to serve as a catalyst for the asymmetric conjugate addition reactions between various thiols and 3-crotonoyl-2-oxazolidinone, affording the corresponding adducts in good yields and high enantioselectivies (up to $92 \%$ ee). A new improved method for making $(4 S, 5 S)$-diphenyl PYBOX is presented.

The Michael addition is widely recognized as one of the most important carbon-carbon bond-forming reactions in organic synthesis. ${ }^{1}$ Several reagent systems for this type of transformation have been developed that rely on chiral catalysts. However, the asymmetric conjugate addition of thiols to acyclic $\alpha, \beta$ unsaturated carbonyl systems has proved to be very challenging.

For thiols, different types of catalysts have been utilized, such as cinchona alkaloids, ${ }^{2}$ chiral proline derivatives, ${ }^{3}$ salens, ${ }^{4}$ $\mathrm{N}$-oxides, ${ }^{5}$ a chiral amino ether-lithium thiolate complex, ${ }^{6}$ and lanthanoid tris(binaphthoxide). ${ }^{7}$ The most effective chiral catalyst so far has been the nickel(II) aqua complex of 4,6-dibenzofurandiyl-2,2'-bis(4-phenyloxazoline) (DBFOX/Ph) ${ }^{8}$ which gives enantioselectivities up to $97 \%$ ee with additions of arylthiols to 3-crotonoyl-2-oxazolidinone. Unfortunately, the catalyst easily degrades under the reaction conditions, as is also reported in the literature, so an improved catalyst system is in demand.

* Corresponding author. Tel.: 358-9-451-2526. Fax: 358-9-451-2538.

(1) Michael, A. J. Prakt. Chem. 1887, 35, 349-356.

(2) Yamashita, H.; Mukaiyama, T. Chem. Lett. 1985, 363-366.

(3) Kobayashi, S.; Ogawa, C.; Kawamura, M.; Sugiura, M. Synlett 2001, 983-985.

(4) Matsumoto, K.; Watanabe, A.; Uchida, T.; Ogi, K.; Katsuki, T. Tetrahedron Lett. 2004, 45, 2385-2388.

(5) Saito, M.; Nakajima, M.; Hashimoto, S. Tetrahedron 2000, 56, 95899594.

(6) Nishimura, K.; Tomioka, K. J. Org. Chem. 2002, 67, 431-434.

(7) Emori, E.; Arai, T.; Sasai, H.; Shibasaki, M. J. Am. Chem. Soc. 1998, 120, 4043-4044.

(8) Kanemasa, S.; Oderaotoshi, Y.; Wada, E. J. Am. Chem. Soc. 1999, $121,8675-8676$.
Previous studies in our group have shown that the 2,6-bis(4'-phenyloxazolin-2'-yl)pyridine ( $\mathrm{Ph}-\mathrm{PYBOX})$ ligand can be used in the asymmetric conjugate addition of thiols, for which asymmetric inductions of up to $67 \%$ ee were obtained. ${ }^{9}$ Desimoni et al. have used $(4 S, 5 S)$-diphenyl PYBOX for Mukaiyama-Michael ${ }^{10}$ and exo-Diels-Alder ${ }^{11}$ reactions with good results. We decided to investigate the activity in the thioMichael addition.

The $(4 S, 5 S)$-diphenyl PYBOX was initially prepared by the method of Desimoni et al., ${ }^{10}$ but the yields in our attempts were low. An improved cyclization with the Burgess reagent is presented in Scheme 1. Ligand $\mathbf{1}$ was applied in the thiol conjugate addition with $\mathrm{Sc}(\mathrm{OTf})_{3}$.

\section{SCHEME 1. Preparation of (4S,5S)-Diphenyl PYBOX}

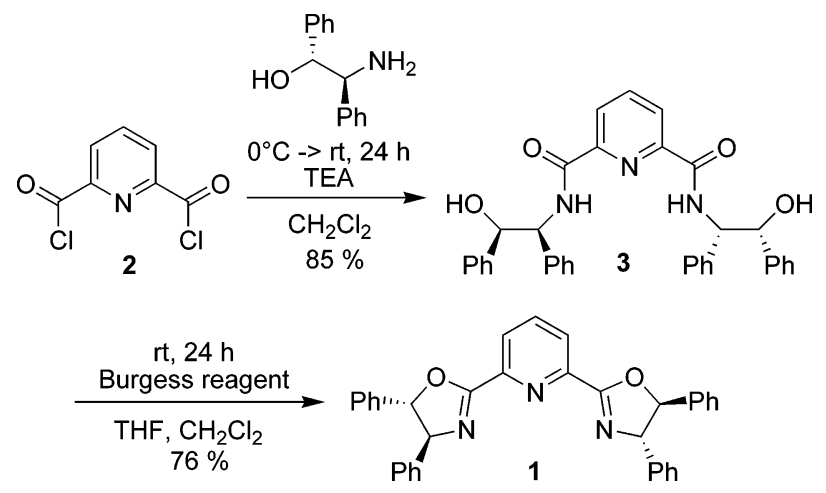

First, the effects of temperature, solvent, and catalyst amount were studied. Reactions were stirred for $24 \mathrm{~h}$. The results obtained are shown in Table 1. Enantioselectivity was improved $7 \%$ when going from room temperature to $0^{\circ} \mathrm{C}$ (Table 1 , entries 3 and 5). There was no change in enantioselectivity when going from 0 to $-12{ }^{\circ} \mathrm{C}$ (Table 1, entries 5 and 6). Apparently, the solubility of the catalyst becomes an issue. Among the tested solvents, the best enantioselectivities were obtained with dichloromethane. Enantioselectivity and yield were improved when $10 \mathrm{~mol} \mathrm{\%}$ of catalyst was used instead of $5 \mathrm{~mol} \mathrm{\%}$ (Table 1 , entries 2 and 3). The unselective background reaction was also monitored. At room temperature, the effect of the background reaction was significant (Table 1 , entry 11 ). When cooled to $0{ }^{\circ} \mathrm{C}$, less than $2 \%$ conversion was observed (Table 1 , entry $10)$.

Having optimized the reaction conditions, we tested the ligand with a variety of thiols and 3-crotonoyl-2-oxazolidinone. ${ }^{12}$ The chiral complex was prepared by reacting $\mathrm{Sc}(\mathrm{OTf})_{3}(10 \mathrm{~mol} \%)$ and $(4 S, 5 S)$-diphenyl PYBOX $1(10 \mathrm{~mol} \%)$ in dichloromethane. 3-Crotonoyl-2-oxazolidinone $4(100 \mathrm{~mol} \%)$ was added at the same time. After $1 \mathrm{~h}$ of stirring at room temperature, the solution was cooled to $0{ }^{\circ} \mathrm{C}$. Thiols $(150 \mathrm{~mol} \%)$ were added. After 20 $\mathrm{h}$ of stirring at $0{ }^{\circ} \mathrm{C}$, the conjugate addition products $\mathbf{5 a}-\mathbf{f}$ were

(9) Sauerland, S. J. K.; Kiljunen, E.; Koskinen, A. M. P. Tetrahedron Lett. 2006, 47, 1291-1293.

(10) Desimoni, G.; Faita, G.; Filippone, S.; Mella, M.; Zampori, M. G.; Zema, M. Tetrahedron 2001, 57, 10203-10212.

(11) Desimoni, G.; Faita, G.; Guala, M.; Pratelli, C. Tetrahedron 2002, 58, 2929-2935.

(12) Other substrates were also tested but with poor results. 3-Cinnamoyloxazolidin-2-one and $(E)$-3-hept-2-enoyloxazolidin-2-one with various thiols yielded only traces or no product. 
TABLE 1. Evaluation of Effects of Catalyst Amount, Solvent, and Temperature

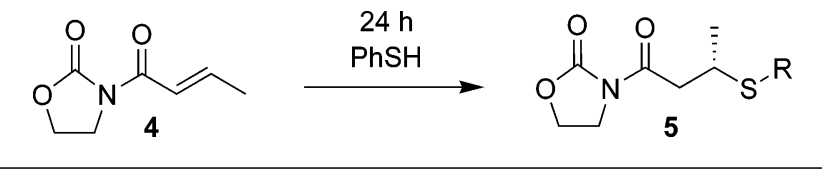

\begin{tabular}{rrlrrc}
\hline entry & cat. mol $\%$ & \multicolumn{1}{c}{ solvent } & temp & yield $\%$ & $\%$ ee \\
\hline 1 & 10 & $\mathrm{THF}$ & $\mathrm{rt}$ & 75 & 66 \\
2 & 5 & $\mathrm{CH}_{2} \mathrm{Cl}_{2}$ & $\mathrm{rt}$ & 77 & 81 \\
3 & 10 & $\mathrm{CH}_{2} \mathrm{Cl}_{2}$ & $\mathrm{rt}$ & 97 & 85 \\
4 & 10 & $\mathrm{CH}_{2} \mathrm{Cl}_{2}$ & $\mathrm{rt}$ & 83 & $70^{a}$ \\
5 & 10 & $\mathrm{CH}_{2} \mathrm{Cl}_{2}$ & 0 & 95 & 92 \\
6 & 10 & $\mathrm{CH}_{2} \mathrm{Cl}_{2}$ & -12 & 93 & 92 \\
7 & 10 & $\mathrm{CHCl}_{3}$ & 0 & 88 & 89 \\
8 & 10 & $\mathrm{MTBE}_{2} \mathrm{CH}_{2} \mathrm{Cl}_{2}$ & 0 & 95 & 89 \\
9 & 10 & $\mathrm{MeCN}_{10}$ & 0 & 82 & 70 \\
10 & 0 & $\mathrm{CH}_{2} \mathrm{Cl}_{2}$ & 0 & $2^{b}$ & 0 \\
11 & 0 & $\mathrm{CH}_{2} \mathrm{Cl}_{2}$ & $\mathrm{rt}$ & $17^{b}$ & 0
\end{tabular}

${ }^{a}$ Proton sponge $(10 \mathrm{~mol} \%)$ was used. ${ }^{b}$ Conversion calculated by integrating the ${ }^{1} \mathrm{H}$ NMR spectrum.

TABLE 2. (4S,5S)-Diphenyl PYBOX (1)-Catalyzed Conjugate Addition

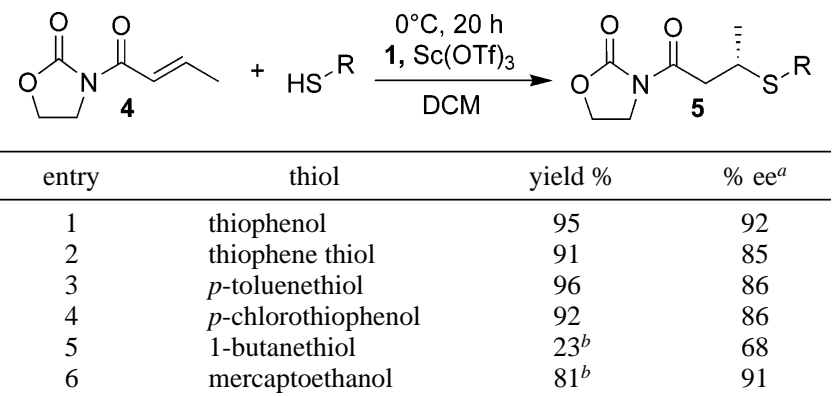

${ }^{a}$ Absolute stereochemistries were established for entries 1 and 3 (ref 8) and 2 (ref 9), others by analogy. ${ }^{b}$ No complete conversion.

then obtained by workup with column chromatography. The $\mathrm{Sc}(\mathrm{III})$ triflate complex of $(4 S, 5 S)$-diphenyl pybox gives products $\mathbf{5 a}$ and $\mathbf{5 b}$ with an absolute configuration $(S)$. With aryl thiols, yields and enantiomeric excesses were high. 1-Butanethiol reacted more slowly than its aromatic counterparts. Erosion in enantioselectivity was also witnessed. The results are summarized in Table 2.

In conclusion, we have demonstrated the effectiveness of the Sc complex of $(4 S, 5 S)$-diphenyl PYBOX for the enantioselective conjugate addition of thiols to 3-crotonoyl-2-oxazolidinone. Further studies on the scope of PYBOX-derived catalysts in asymmetric Michael addition of thiols are currently underway.

\section{Experimental Section}

$N^{2}, N^{6}$-Bis((1S,2S)-2-hydroxy-1,2-diphenylethyl)pyridine-2,6dicarboxamide (3). $(1 R, 2 S)$-2-Amino-1,2-diphenylethanol $(2.1 \mathrm{~g}$, $10.0 \mathrm{mmol}, 200 \mathrm{~mol} \%)$ and triethylamine $(3.4 \mathrm{~mL}, 24.5 \mathrm{mmol}$, $490 \mathrm{~mol} \%)$ were dissolved in $\mathrm{CH}_{2} \mathrm{Cl}_{2}(35 \mathrm{~mL})$, and the mixture was cooled to $0{ }^{\circ} \mathrm{C}$. 2,6-Pyridinedicarbonylchloride $(1.0 \mathrm{~g}, 5.0$ mmol, $100 \mathrm{~mol} \%$ ) was dissolved in $\mathrm{CH}_{2} \mathrm{Cl}_{2}(15 \mathrm{~mL})$ and added to the reaction mixture via cannula. The cooling bath was removed, and the solution was stirred for $20 \mathrm{~h}$ at room temperature. The reaction mixture was filtered, and the solid was washed with water 4 times. The product was collected to yield $3(2.6 \mathrm{~g}, 92 \%)$ as a white solid. ${ }^{1} \mathrm{H} \mathrm{NMR}\left(400 \mathrm{MHz}, \mathrm{CDCl}_{3}\right) \delta 8.99(\mathrm{~d}, 2 \mathrm{H}, J=8.8$ $\mathrm{Hz}), 8.12-8.04(\mathrm{~m}, 3 \mathrm{H}), 7.42-7.14(\mathrm{~m}, 20 \mathrm{H}), 5.84$ (d, 2H, $J=$ $4.8 \mathrm{~Hz}), 5.25-5.14(\mathrm{~m}, 4 \mathrm{H}){ }^{10}$
2,6-Bis $((4 S, 5 S)-4,5$-diphenyl-4,5-dihydrooxazol-2-yl)pyridine (1). Amidoalcohol 3 (1.44 g, $2.6 \mathrm{mmol}, 100 \mathrm{~mol} \%)$ was suspended in THF $(80 \mathrm{~mL})$ and $\mathrm{CH}_{2} \mathrm{Cl}_{2}(25 \mathrm{~mL})$. Burgess reagent (1.8 g, $7.6 \mathrm{mmol}, 290 \mathrm{~mol} \%$ ) was added. The solid dissolved in $20 \mathrm{~min}$. The reaction mixture was stirred for $17 \mathrm{~h}$ at room temperature, after which time the solvents were evaporated. The crude product was crystallized from ethyl acetate. The product was collected to yield $\mathbf{1}(1.02 \mathrm{~g}, 76 \%)$ as colorless crystals. ${ }^{1} \mathrm{H}$ NMR $\left(400 \mathrm{MHz}, \mathrm{CDCl}_{3}\right) \delta 8.39(\mathrm{~d}, 2 \mathrm{H}, J=7.9 \mathrm{~Hz}), 7.98(\mathrm{t}, 1 \mathrm{H}, J=$ $7.9 \mathrm{~Hz}), 7.33-7.26(\mathrm{~m}, 20 \mathrm{H}), 5.53(\mathrm{~d}, 2 \mathrm{H}, J=8.4 \mathrm{~Hz}), 5.33(\mathrm{~d}$, $2 \mathrm{H}, J=8.4 \mathrm{~Hz}){ }^{10}$

General Procedure for the Asymmetric Conjugate Addition: (S)-3-(3-(Phenylthio)butanoyl)oxazolidin-2-one (5a). 2,6$\operatorname{Bis}((4 S, 5 S)-4,5$-diphenyl-4,5-dihydrooxazol-2-yl)pyridine (16 mg, $0.03 \mathrm{mmol}, 10 \mathrm{~mol} \%)$, Sc(OTf) 3 (15 mg, $0.03 \mathrm{mmol}, 10 \mathrm{~mol} \%)$, and crotonoyl-oxazolidinone (47 $\mathrm{mg}, 0.3 \mathrm{mmol}, 100 \mathrm{~mol} \%$ ) were dissolved in $\mathrm{CH}_{2} \mathrm{Cl}_{2}(1.2 \mathrm{~mL})$. The solution was stirred for $1 \mathrm{~h}$ at room temperature. The mixture was then cooled to $0{ }^{\circ} \mathrm{C}$, and thiophenol ( $48 \mu \mathrm{L}, 0.45 \mathrm{mmol}, 150 \mathrm{~mol} \%$ ) was added. After stirring for $20 \mathrm{~h}$ at $0{ }^{\circ} \mathrm{C}$, the products were isolated by adding the cold reaction mixture directly to a silica gel column and eluting with a $\mathrm{CH}_{2} \mathrm{Cl}_{2} / \mathrm{MeOH}$ solvent mixture. The product was collected to yield 5a $(75 \mathrm{mg}, 95 \%)$ as a colorless oil; $R_{\mathrm{f}}=0.72$ in $1: 30 \mathrm{MeOH} / \mathrm{CH}_{2-}$ $\mathrm{Cl}_{2} ; \mathrm{HPLC}^{13} R_{\mathrm{t}}^{\text {major }}=40.6 \mathrm{~min}, R_{\mathrm{t}}^{\text {minor }}=34.2 \mathrm{~min}$, ee $=92 \%$; $[\alpha]_{\mathrm{D}}{ }^{20}=-28\left(c 1.0, \mathrm{CH}_{2} \mathrm{Cl}_{2}\right) ;{ }^{1} \mathrm{H}$ NMR $\left(400 \mathrm{MHz}, \mathrm{CDCl}_{3}\right) \delta 7.45$ $(\mathrm{d}, 2 \mathrm{H}, J=7.0 \mathrm{~Hz}), 7.32-7.22(\mathrm{~m}, 3 \mathrm{H}), 4.36(\mathrm{t}, 2 \mathrm{H}, J=8.0 \mathrm{~Hz})$, $3.94(\mathrm{t}, 2 \mathrm{H}, J=8.0 \mathrm{~Hz}), 3.77(\mathrm{ddq}, 1 \mathrm{H}, J=6.8 \mathrm{~Hz}, 6.8 \mathrm{~Hz}, 6.8$ $\mathrm{Hz}), 3.26(\mathrm{dd}, 1 \mathrm{H}, J=7.0 \mathrm{~Hz}, 17.0 \mathrm{~Hz}), 3.13(\mathrm{dd}, 1 \mathrm{H}, J=7.0 \mathrm{~Hz}$, $17.0 \mathrm{~Hz}), 1.36(\mathrm{~d}, 3 \mathrm{H}, J=6.8 \mathrm{~Hz}){ }^{9}$

(S)-3-(3-(Thiophen-2-ylthio)butanoyl)-oxazolidin-2-one (5b). Yield $91 \%$ (slightly yellow oil); $R_{\mathrm{f}}=0.78$ in $1: 30 \mathrm{MeOH} / \mathrm{CH}_{2} \mathrm{Cl}_{2}$; $\mathrm{HPLC}^{14} R_{\mathrm{t}}^{\text {major }}=59.4 \mathrm{~min}, R_{\mathrm{t}}^{\text {minor }}=42.2 \mathrm{~min}$, ee $=85 \%$; $[\alpha]_{\mathrm{D}}{ }^{20}$ $=-7\left(c 0.3, \mathrm{CH}_{2} \mathrm{Cl}_{2}\right) ;{ }^{1} \mathrm{H} \mathrm{NMR}\left(400 \mathrm{MHz}, \mathrm{CDCl}_{3}\right) \delta 7.40(\mathrm{dd}$, $1 \mathrm{H}, J=1.2 \mathrm{~Hz}, 5.4 \mathrm{~Hz}), 7.18(\mathrm{dd}, 1 \mathrm{H}, J=1.2 \mathrm{~Hz}, 3.5 \mathrm{~Hz}), 7.01$ $(\mathrm{dd}, 1 \mathrm{H}, J=3.5 \mathrm{~Hz}, 5.4 \mathrm{~Hz}), 4.41(\mathrm{t}, 2 \mathrm{H}, J=7.9 \mathrm{~Hz}), 4.01(\mathrm{t}, 2 \mathrm{H}$, $J=7.9 \mathrm{~Hz}), 3.53(\mathrm{ddq}, 1 \mathrm{H}, J=6.8 \mathrm{~Hz}, 6.8 \mathrm{~Hz}, 6.8 \mathrm{~Hz}), 3.33(\mathrm{dd}$, $1 \mathrm{H}, J=6.8 \mathrm{~Hz}, 17.3 \mathrm{~Hz}), 3.07(\mathrm{dd}, 1 \mathrm{H}, J=6.8 \mathrm{~Hz}, 17.3 \mathrm{~Hz})$, 1.34. (d, $3 \mathrm{H}, J=6.8 \mathrm{~Hz}){ }^{9}$

(S)-3-(3-(p-Tolylthio)butanoyl)-oxazolidin-2-one (5c). Yield $96 \%$ (colorless oil); $R_{\mathrm{f}}=0.76$ in $1: 30 \mathrm{MeOH} / \mathrm{CH}_{2} \mathrm{Cl}_{2} ; \mathrm{HPLC}^{13}$ $R_{\mathrm{t}}^{\text {major }}=35.2 \mathrm{~min}, R_{\mathrm{t}}^{\text {minor }}=29.1 \mathrm{~min}$, ee $=86 \% ;[\alpha]_{\mathrm{D}}^{20}=-22(c$ 1.0, $\left.\mathrm{CH}_{2} \mathrm{Cl}_{2}\right) ;{ }^{1} \mathrm{H}$ NMR $\left(400 \mathrm{MHz}, \mathrm{CDCl}_{3}\right) \delta 7.34(\mathrm{~d}, 2 \mathrm{H}, J=8.0$ $\mathrm{Hz}), 7.12(\mathrm{~d}, 2 \mathrm{H}, J=8.0 \mathrm{~Hz}), 4.37(\mathrm{t}, 2 \mathrm{H}, J=8.0 \mathrm{~Hz}), 3.96(\mathrm{t}$, $2 \mathrm{H}, J=8.0 \mathrm{~Hz}), 3.69(\mathrm{ddq}, 1 \mathrm{H}, J=6.8 \mathrm{~Hz}, 6.8 \mathrm{~Hz}, 6.8 \mathrm{~Hz}), 3.25$ $(\mathrm{dd}, 1 \mathrm{H}, J=6.8 \mathrm{~Hz}, 17.0 \mathrm{~Hz}), 3.10(\mathrm{dd}, 1 \mathrm{H}, J=6.8 \mathrm{~Hz}, 17.0 \mathrm{~Hz})$, $2.33(\mathrm{~s}, 3 \mathrm{H}), 1.33 .(\mathrm{d}, 3 \mathrm{H}, J=6.8 \mathrm{~Hz}){ }^{10}$

(S)-3-(3-(4-Chlorophenylthio)butanoyl)oxazolidin-2-one (5d). Yield $92 \%$ (colorless oil); $R_{\mathrm{f}}=0.74$ in $1: 30 \mathrm{MeOH} / \mathrm{CH}_{2} \mathrm{Cl}_{2}$; $\mathrm{HPLC}^{13} R_{\mathrm{t}}^{\text {major }}=38.9 \min , R_{\mathrm{t}}^{\text {minor }}=35.4 \mathrm{~min}$, ee $=86 \%$; $[\alpha]_{\mathrm{D}}{ }^{20}$ $=-21$ (c 1.0, $\left.\mathrm{CH}_{2} \mathrm{Cl}_{2}\right)$; IR $\left(\mathrm{CH}_{2} \mathrm{Cl}_{2}, \mathrm{~cm}^{-1}\right)$ : 3064, 2970, 2927, 2871, 1783, 1702, 1477, 1387. ${ }^{1} \mathrm{H}$ NMR (400 MHz, $\left.\mathrm{CDCl}_{3}\right) \delta 7.39$ $(\mathrm{d}, 2 \mathrm{H}, J=8.5 \mathrm{~Hz}), 7.28(\mathrm{~d}, 2 \mathrm{H}, J=8.5 \mathrm{~Hz}), 4.39$ (t, $2 \mathrm{H}, J=8.1$ $\mathrm{Hz}), 3.97$ (t, $2 \mathrm{H}, J=8.1 \mathrm{~Hz}), 3.73(\mathrm{ddq}, 1 \mathrm{H}, J=6.8 \mathrm{~Hz}, 6.8 \mathrm{~Hz}$, $6.8 \mathrm{~Hz}), 3.26(\mathrm{dd}, 1 \mathrm{H}, J=6.8 \mathrm{~Hz}, 17.1 \mathrm{~Hz}), 3.11(\mathrm{dd}, 1 \mathrm{H}, J=6.8$ $\mathrm{Hz}, 17.1 \mathrm{~Hz}), 1.35 .(\mathrm{d}, 3 \mathrm{H}, J=6.8 \mathrm{~Hz}) \cdot{ }^{13} \mathrm{C}$ NMR $(100 \mathrm{MHz}$, $\left.\mathrm{CDCl}_{3}\right) \delta 170.9,153.3,134.2,133.6,132.5,129.0,62.1,42.4,42.2$, 39.2 , 21.2. HRMS (ESI) calcd for $\mathrm{C}_{13} \mathrm{H}_{14} \mathrm{NO}_{3} \mathrm{NaSCl}[\mathrm{M}+\mathrm{Na}]^{+}$ 322.0269, found 322.0281.

(S)-3-(3-(Butylthio)butanoyl)-oxazolidin-2-one (5e). Yield 66\% (colorless foam); $R_{\mathrm{f}}=0.63$ in 1:30 MeOH/CH $\mathrm{CH}_{2} ; \mathrm{HPLC}^{13} R_{\mathrm{t}}$ major $=24.9 \mathrm{~min}, R_{\mathrm{t}}^{\text {minor }}=22.6 \mathrm{~min}$, ee $=68 \% ;[\alpha]_{\mathrm{D}}{ }^{20}=-1$ (c 1.0, $\left.\mathrm{CH}_{2} \mathrm{Cl}_{2}\right)$; IR $\left(\mathrm{CH}_{2} \mathrm{Cl}_{2}, \mathrm{~cm}^{-1}\right)$ : 3062, 2962, 2929, 2874, 1782, 1702, 1386. ${ }^{1} \mathrm{H} \mathrm{NMR}\left(400 \mathrm{MHz}, \mathrm{CDCl}_{3}\right) \delta 7.44(\mathrm{t}, 2 \mathrm{H}, J=8.1 \mathrm{~Hz})$, $4.34(\mathrm{t}, 2 \mathrm{H}, J=8.1 \mathrm{~Hz}), 3.97(\mathrm{t}, 2 \mathrm{H}, J=8.1 \mathrm{~Hz}), 3.25(\mathrm{~m}, 1 \mathrm{H})$, $2.97(\mathrm{~m}, 1 \mathrm{H}), 2.51(\mathrm{t}, 2 \mathrm{H}, J=7.3 \mathrm{~Hz}), 1.5(\mathrm{tt}, 2 \mathrm{H}, J=7.3 \mathrm{~Hz}, 7.3$

(13) HPLC analysis was performed on a Daicel OD column eluting with 10\% 2-propanol/hexanes. 
$\mathrm{Hz}), 1.34(\mathrm{tq}, 2 \mathrm{H}, J=7.3 \mathrm{~Hz}, 7.3 \mathrm{~Hz}), 1.29(\mathrm{~d}, 3 \mathrm{H}, J=6.8 \mathrm{~Hz})$, $0.84(\mathrm{t}, 3 \mathrm{H}, J=7.3 \mathrm{~Hz}) .{ }^{13} \mathrm{C}$ NMR $\left(100 \mathrm{MHz}, \mathrm{CDCl}_{3}\right) \delta 171.2$, 153.4, 62.0, 42.6, 42.5, 35.5, 31.8, 30.22, 22.1, 21.7, 13.7. HRMS (ESI) calcd for $\mathrm{C}_{11} \mathrm{H}_{19} \mathrm{NO}_{3} \mathrm{NaS}[\mathrm{M}+\mathrm{Na}]^{+}$268.0983, found 268.0986.

(S)-(3-(2-Hydroxyethylthio)butanoyl)-oxazolidin-2-one (5f). Yield $81 \%$ (colorless oil); $R_{\mathrm{f}}=0.56$ in $1: 30 \mathrm{MeOH} / \mathrm{CH}_{2} \mathrm{Cl}_{2}$; $\mathrm{HPLC}^{13} R_{\mathrm{t}}^{\text {major }}=32.2 \mathrm{~min}, R_{\mathrm{t}}^{\text {minor }}=26.3 \mathrm{~min}$, ee $=91 \% ;[\alpha]_{\mathrm{D}}^{20}$ $=-4\left(c 0.3, \mathrm{CH}_{2} \mathrm{Cl}_{2}\right)$; IR $\left(\mathrm{CH}_{2} \mathrm{Cl}_{2}, \mathrm{~cm}^{-1}\right): 3609,3463,3056,2966$, 2926, 2874, 1782, 1701, 1387. ${ }^{1} \mathrm{H}$ NMR (400 MHz, $\left.\mathrm{CDCl}_{3}\right) \delta 4.44$ $(\mathrm{t}, 1 \mathrm{H}, J=8.1 \mathrm{~Hz}), 4.05(\mathrm{dt}, 2 \mathrm{H}, J=2.1 \mathrm{~Hz}, 7.9 \mathrm{~Hz}), 3.77(\mathrm{~m}$, $2 \mathrm{H}), 3.34(\mathrm{dd}, 2 \mathrm{H}, J=6.6 \mathrm{~Hz}, 14.0 \mathrm{~Hz}), 3.03(\mathrm{q}, 1 \mathrm{H}, J=8.9 \mathrm{~Hz})$, $2.79(\mathrm{dt}, 2 \mathrm{H}, J=1.3 \mathrm{~Hz}, 6.0 \mathrm{~Hz}), 1.39(\mathrm{~d}, 3 \mathrm{H}, J=6.7 \mathrm{~Hz}) .{ }^{13} \mathrm{C}$
NMR (100 MHz, $\left.\mathrm{CDCl}_{3}\right) \delta 171.2,153.4,62.1,61.1,42.5,42.4$, 35.3, 33.8, 22.0. HRMS (ESI) calcd for $\mathrm{C}_{9} \mathrm{H}_{15} \mathrm{NO}_{4} \mathrm{NaS}[\mathrm{M}+\mathrm{Na}]^{+}$ 256.0619, found 256.0608 .

Acknowledgment. Financial support from PCAS Finland is gratefully acknowledged. We also thank Anna-Maija Horko, Esa Kumpulainen, and Dr. Jari Koivisto for assistance.

Supporting Information Available: Characterization for compounds $\mathbf{1}$ and $\mathbf{5 a}-\mathbf{f}$. This material is available free of charge via the Internet at http://pubs.acs.org.

JO070492Z 